Pacific Journal of Mathematics

CENTROID SURFACES 


\title{
CENTROID SURFACES
}

\author{
C. M. Petty
}

1. Introduction. Let $M_{1}, \cdots, M_{n-1}$ denote $(n-1)$ bounded closed sets in $E_{n}$. Busemann [1] has established the expression

$$
\begin{aligned}
& \left|M_{1}\right| \cdots\left|M_{n-1}\right|= \\
& \quad \frac{(n-1) !}{2} \int_{\Omega_{n}}\left(\int_{M_{1}(u)} \cdots \int_{M_{n-1}(u)} T\left(z, p_{1}, \cdots, p_{n-1}\right) d V_{p_{1}}^{n-1} \cdots d V_{p_{n-1}}^{n-1}\right) d \omega_{u}^{n}
\end{aligned}
$$

where $\left|M_{i}\right|$ is the $n$-dimensional Lebesgue measure or volume of $M_{i}$. On the righthand side $M_{i}(u)$ is the cross-section of $M_{i}$ with the hyperplane through $z$ normal to the unit vector $u$, the point $p_{i}$ varies in $M_{i}(u)$ and the differential $d V_{p_{i}}^{n-1}$ is the $(n-1)$-dimensional volume element of $M_{i}(u)$ at $p_{i}$. The final integration is extended over the surface $\Omega_{n}$ of the solid-unit sphere $U_{n}$ and $d \omega_{u}^{n}$ is the area element of $\Omega_{n}$ at point $u$. By $T\left(z, p_{1}, \cdots, p_{r}\right)$ we will denote the $r$-dimensional volume of the simplex (possibly degenerate) with vertices $z, p_{1}, \cdots, p_{r}$.

Let

$$
\pi_{r}=\frac{\pi^{r / 2}}{\Gamma(r / 2+1)} .
$$

For $n \geqq 3$, Busemann also shows by Steiner's symmetrization that

$$
\left|M_{1}\right| \cdots\left|M_{n-1}\right| \geqq \frac{1}{n} \frac{\pi_{n}^{n-2}}{\pi_{n-1}^{n}} \int_{\Omega_{n}}\left|M_{1}(u)\right|^{n /(n-1)} \cdots\left|M_{n-1}(u)\right|^{n /(n-1)} d \omega_{u}^{n}
$$

for nondegenerate convex bodies $M_{i}$ where the equality sign holds only when the $M_{i}$ are homothetic solid ellipsoids with center $z$. Here $\left|M_{i}(u)\right|$, of course, denotes the $(n-1)$-dimensional volume of $M_{i}(u)$. In this regard we will also, as a matter of convenience, not index lower dimensional mixed discriminates and mixed volumes since the dimension will be evident from the number of components.

The primary purpose of this note is to reinterpret (1.1) as an integration of the type (1.3) retaining the equality sign. This is given in $\S 3$ by (3.20). In addition other integral expressions and inequalities are derived which are geometrically of the same type as those considered above.

2. Fenchel's momental ellipsoid. Let $M$ be a bounded closed set with positive volume. The centroid $s$ of $M$ is defined by its rectangular coordinates

Received January 3, 1961. 


$$
s_{i}=\frac{1}{|M|} \int_{M} x_{i} d V_{x}^{n}
$$

If $L_{\nu}$ is a $\nu$-flat through the origin $z$, then the second moment of $M$ with respect to $L_{\nu}(0 \leqq \nu \leqq n-1)$ is defined by

$$
I\left(M, L_{\nu}\right)=\int_{M} r^{2} \sin ^{2} \varphi d V_{x}^{n}
$$

where the distance $z x$ is $r$ and $\varphi$ is the angle between the ray $z x$ and $L_{\nu}$ (for $\nu=0$, we define $\varphi=\pi / 2$ ). By the same type of integration technique in $[1, \mathrm{pp} .5-6]$, the reader may verify that

$$
I\left(U_{n}, L_{\nu}\right)=\frac{n-\nu}{n+2} \pi_{n}
$$

where $U_{n}$ has center $z$; a calculation which will be used later.

The matrix $A_{M}$ given by

$$
A_{M}=\left[\frac{1}{|M|} \int_{M} x_{i} x_{j} d V_{x}^{n}\right]
$$

is positive definite since

$$
y^{T} A_{M} y=\frac{1}{|M|} \int_{M}\left(\Sigma x_{i} y_{i}\right)^{2} d V_{x}^{n}
$$

where $y$ is a column vector and $y^{T}$ is its transpose. The ellipsoid with surface $x^{T} A_{M} x=1$ will be called Fenchel's momental ellipsoid and its polar reciprocal with respect to $\Omega_{n}$ given by $x^{T} A_{M}^{-1} x=1$ will be called simply Fenchel's ellipsoid. This name is chosen since $W$. Fenchel first observed the affine character of this polar reciprocal (unpublished):

(2.5) Let $M$ be transformed into $\bar{M}$ by a central affinity with matrix $B$. If $F$ and $\bar{F}$ are the Fenchel ellipsoids of $M$ and $\bar{M}$ respectively, then this central affinity also carries $F$ into $\bar{F}$.

To see this, it may be observed from (2.4) that $A_{\bar{M}}=B A_{\mu} B^{r}$ or $A_{\overline{\mathbf{u}}}^{-1}=\left(B^{-1}\right)^{T} A_{\dot{M}}^{-1} B^{-1}$ which completes the proof.

If $|F|$ is the volume of the Fenchel ellipsoid $F$ of $M$, then

$$
|F|^{2}=\pi_{n}^{2} \operatorname{det}\left(A_{M}\right) \text {. }
$$

The result (2.5) enables one to prove readily that

$$
\pi_{n}^{-2}|F|^{2}=\operatorname{det}\left(A_{M}\right) \geqq(n+2)^{-n} \pi_{n}^{-2}|M|^{2}
$$

with equality only if, except for a set of measure zero, $M$ is a solid ellipsoid with center $z$. For if we transform $M$ into $\bar{M}$ by a unimodular central affinity so that $\bar{F}$ is a sphere, then 


$$
\operatorname{det}\left(A_{M}\right)=\left[\frac{1}{n|M|} \int_{\bar{M}} r^{2} d V_{x}^{n}\right]^{n}
$$

Comparison of $\int_{\bar{M}} r^{2} d V_{x}^{n}$ with that for a sphere with center $z$ and volume $|M|$ proves (2.7).

We will adopt the same notation for mixed discriminates as in [2, pp. 51-57] where the reader will find an exposition of their properties. Consider the $r$ quadratic forms $q_{i}=x^{T} A_{i} x, i=1, \cdots, r$, where $A_{k}=$ $\left[a_{i j}^{(k)}\right]$ is a real symmetric matrix. For any real $\lambda_{1}, \cdots, \lambda_{r}$, set $q=\lambda_{1} q_{1}+$ $\cdots+\lambda_{r} q_{r}=x^{r} A x$ where $A=\sum_{k=1}^{r} \lambda_{k} A_{k}$. The discriminant $D(q)=\operatorname{det}(A)$ can be written

$$
D(q)=\sum_{i_{1}=1}^{r} \cdots \sum_{i_{n}=1}^{r} \lambda_{i_{1}} \cdots \lambda_{i_{n}} D\left(q_{i_{1}}, \cdots, q_{i_{n}}\right)
$$

where $D\left(q_{i_{1}}, \cdots, q_{i_{n}}\right)$ is independent of the order of the $q_{i_{k}}$ and is called the mixed discriminant of $q_{i_{1}}, \cdots, q_{i_{n}}$. For $n$ forms $q_{i}$ we have

$$
D\left(q_{1}, \cdots, q_{n}\right)=\frac{1}{n !} \sum_{\left(i_{1} \cdots v_{n}\right)}\left|\begin{array}{ccc}
a_{11}^{\left(i_{1}\right)} & \cdots & a_{1 n}^{\left(i_{n}\right)} \\
\vdots & & \vdots \\
a_{n 1}^{\left(i_{1}\right)} & \cdots & a_{n n}^{\left(i_{n}\right)}
\end{array}\right|
$$

where $\left(i_{1} \cdots i_{n}\right)$ is a permutation of $(1 \cdots n)$.

Now consider $n$ closed and bounded sets $M_{i}$ with positive volume and let $q_{i}=x^{T} A_{\alpha_{i}} x$ be the quadratic form associated with the Fenchel momental ellipsoid of $M_{i}$. By (2.4) and (2.8) we have

$$
\begin{aligned}
& D\left(q_{1}, \cdots, q_{n}\right)=\frac{1}{n !\left|M_{1}\right| \cdots\left|M_{n}\right|} \\
& \sum_{\left(i_{1} \ldots n_{n}\right)} \int_{M_{i_{1}}} \cdots \int_{M_{i_{n}}{ }_{n}} x_{1}^{\left(i_{1}\right)} \cdots x_{n}^{\left(i_{n}\right)}\left|\begin{array}{ccc}
x_{1}^{\left(i_{1}\right)} & \cdots & x_{1}^{\left(i_{n}\right)} \\
\vdots & & \vdots \\
x_{n}^{\left(i_{1}\right)} & \cdots & x_{n}^{\left(i_{n}\right)}
\end{array}\right| d V_{x}^{n}{ }_{x}^{\left(i_{1}\right)} \cdots d V_{x}^{n}{ }_{x}^{\left(i_{n}\right)} \\
& =\frac{1}{n !\left|M_{1}\right| \cdots\left|M_{n}\right|} \int_{M 1} \cdots \int_{M_{n}}\left|\begin{array}{ccc}
x_{1}^{(1)} & \cdots & x_{1}^{(n)} \\
\vdots & \vdots \\
x_{n}^{(1)} & \cdots & x_{n}^{(n)}
\end{array}\right|^{2} d V_{x^{(1)}}^{n} \cdots d V_{x^{(n)}}^{n} .
\end{aligned}
$$

Since $T\left(z, x^{(1)}, \cdots, x^{(n)}\right)= \pm(1 / n !) \operatorname{det}\left(x_{i}^{(j)}\right)$ we then have

$$
\begin{aligned}
& D\left(q_{1}, \cdots, q_{n}\right)= \\
& \quad \frac{n !}{\left|M_{1}\right| \cdots\left|M_{n}\right|} \int_{M_{1}} \cdots \int_{M_{n}} T^{2}\left(z, p_{1}, \cdots, p_{n}\right) d V_{p_{1}}^{n} \cdots d V_{p_{n}}^{n} .
\end{aligned}
$$

The fundamental inequality for mixed discriminants (see [2, p. 53]) is: (2.11) If the forms $q_{1}, \cdots, q_{n-1}$ are positive definite and $Q$ is any sym- 
metric form, then

$$
D^{2}\left(q_{1}, \cdots, q_{n-1}, Q\right) \geqq D\left(q_{1}, \cdots, q_{n-1}, q_{n-1} D\left(q_{1}, \cdots, q_{n-2}, Q, Q\right)\right.
$$

where the equality sign holds only if $Q=\lambda q_{n-1}$.

If we set

$$
D_{p}(q, Q)=D(q_{1}, \cdots, q_{n-p}, \underbrace{Q, \cdots, Q)}_{p},
$$

then for $n$ positive definite forms $q_{i}$, (2.11) generalizes to

$$
D^{r}\left(q_{1}, \cdots, q_{n}\right) \geqq \prod_{k=0}^{r-1} D_{r}\left(q, q_{n-k}\right), r=2,3, \cdots, n
$$

with equality only if $q_{n-k}=\lambda_{n-k} q_{n}$ for $k=0, \cdots, r-1$.

The proof of (2.13) and the condition for equality proceed by induction from the case $r=2$. The proof is analogous to Alexandrov's generalization [2, p. 50] of a corresponding inequality for mixed volumes and consequently will be omitted here.

If we now set

$$
W\left(M_{1}, \cdots, M_{n}, z\right)=\int_{M_{1}} \cdots \int_{M_{n}} T^{2}\left(z, p_{1}, \cdots, p_{n}\right) d V_{p_{1}}^{n} \cdots d V_{p_{n}}^{n}
$$

and

$$
W_{p}\left(M, M_{k}, z\right)=W(M_{1}, \cdots, M_{n-p}, \underbrace{M_{k}, \cdots M_{k}}_{p}, z),
$$

then by (2.13) and (2.10) we have

$$
W^{r}\left(M_{1}, \cdots, M_{n}, z\right) \geqq \prod_{k=0}^{r-1} W_{r}\left(M, M_{n-k}, z\right), r=2, \cdots, n
$$

with the equality sign only if the Fenchel ellipsoids of $M_{n-k}$ are homothetic for $k=0, \cdots, r-1$. Applying (2.16) to the case $r=n$ and using (2.10) and (2.7), we have

$$
\left[\left|M_{1}\right| \cdots\left|M_{n}\right|\right]^{(n+2) / n} \leqq n ! \pi_{n}^{2}(n+2)^{n} W\left(M_{1}, \cdots, M_{n}, z\right)
$$

with equality only if (except for a set of measure zero) the $M_{i}$ are homothetic ellipsoids with center $z$.

The reader will find other inequalities of the above type in [3, pp. $70-71]$.

3. Centroid surfaces. As before, $M$ is a bounded closed set with positive volume. An oriented hyperplane $L(u)$ through $z$ normal to the direction $u(u \neq 0)$ bounds a closed half-space lying on its positive side. 
The intersection of this halfspace with $M$ will be denoted by $C(u)$. Consider the function

$$
H(u)=\frac{1}{|M|} \int_{\boldsymbol{M}}|u \cdot x| d V_{x}^{n}, u \cdot x=\sum_{i=1}^{n} u_{i} x_{i} .
$$

Since

(a) $H(0)=0$,

(b) $H(\mu u)=\mu H(u)$ for $\mu>0$,

(c) $H(u+v) \leqq H(u)+H(v)$,

$H(u)$ is the supporting function (s.f.) of a convex body $K^{*}$ (see $[4, \mathrm{p}$. 26]), which is nondegenerate and has center $z$. Let $P_{0}$ be the supporting plane (s.p.) to $K^{*}$ in the direction $u^{(0)}$, the supporting function of $K^{*} \cap P_{0}$ is given by the directional derivative

$$
\begin{aligned}
H^{\prime}\left(u^{(0)} ; u\right) & =\lim _{h \rightarrow 0+} \frac{H\left(u^{(0)}+h u\right)-H\left(u^{(0)}\right)}{h} \\
& =\frac{1}{|M|} \int_{O\left(u^{(0))}\right.} u \cdot x d V_{x}^{n}-\frac{1}{|M|} \int_{\sigma_{\left(-u^{(0)}\right)}} u \cdot x d V_{x}^{n} .
\end{aligned}
$$

Since $H^{\prime}\left(u^{(0)} ; u\right)$ is a linear function of the $u_{i}, P_{0}$ touches $K^{*}$ in a single point and thus every s.p. of $K^{*}$ is regular and $K^{*}$ is strictly convex. (See [4, pp. 25-26].) The derivatives $\partial H / \partial u_{i}$ are continuous, homogeneous of degree 0 , and if $y$ is the point of contact of the s.p. to $K^{*}$ in the direction $u$, then

$$
y_{i}=\frac{\partial H}{\partial u_{i}}=\frac{1}{|M|} \int_{\sigma(u)} x_{i} d V_{x}^{n}-\frac{1}{|M|} \int_{\sigma(-u)} x_{i} d V_{x}^{n} .
$$

We will call $K^{*}$ the centroid body of $M$ (with respect to $z$ ) and the surface of $K^{*}$ will be called the centroid surface of $M$. One may observe that if $M$ happens to have center $z$, then the centroid surface of $M$ is precisely the set of all controids of $C(u)$ for $u \in \Omega_{n}$. In general, let $s^{(1)}$ and $s^{(2)}$ be the centroids of $C(u)$ and $C(-u)$ respectively, the $y$ is the center of mass of the two points $s^{(1)}$ and $-s^{(2)}$ provided with mass $|C(u)| /|M|$ and $|C(-u)| /|M|$ respectively. If $|C(u)|=0$, we will define the centroid of $C(u)$ to be the point $z$.

It is evident that if $M$ is transformed into $\bar{M}$ by a central affinity, then this transformation also carries the centroid surface of $M$ into the centroid surface of $\bar{M}$.

We now wish to impose additional restrictions on $M$ such that $H(u)$ has continuous second partial derivatives and the surface of $K^{*}$ has positive Gauss curvature. The following two conditions are sufficient for this purpose: 
(a) The set $M(u)$ has positive $(n-1)$-dimensional measure for all $u \in \Omega_{n}$.

(b) For any $u^{(0)} \in \Omega_{n}$ and any sequence $u^{(i)} \rightarrow u^{(0)}$, the $\lim _{u(i) \rightarrow u^{(0)}} M\left(u^{(i)}\right)$ coincides with $M\left(u^{(0)}\right)$ except for a possible set of zero $(n-1)$-dimensional measure.

To simplify the calculation of the second partial derivatives at a point $u^{(0)}$, we introduce what Busemann [2, p. 57] calls "standard coordinates." With the same origin and orientation, the $x_{n}$ axis is chosen such that $u_{1}^{(0)}=\cdots=u_{n-1}^{(0)}=0$ and $u_{n}^{(0)}>0$. It then follows from (3.3) that

$$
\frac{\partial^{2} H\left(u^{(0)}\right)}{\partial u_{k} \partial u_{n}}=\frac{\partial^{2} H\left(u^{(0)}\right)}{\partial u_{n} \partial u_{k}}=0 .
$$

Although standard coordinates vary from point to point, the end result (3.9) is expressed geometrically and therefore independent of the coordinate system.

For $j<n$, let $u=\left(0, \cdots, 0, u_{j}, 0, \cdots, 0, u_{n}^{(0)}\right)$ and set

$$
\begin{aligned}
N_{1} & =C(u) \cap C\left(u^{(0)}\right), N_{1}^{*}=C(-u) \cap C\left(-u^{(0)}\right), N_{2}=C\left(u^{(0)}\right)-N_{1}, \\
N_{2}^{*} & =C(-u)-N_{1}^{*}, \quad N_{3}=C(u)-N_{1}, \quad N_{3}^{*}=C\left(-u^{(0)}\right)-N_{1}^{*} .
\end{aligned}
$$

Except for a set of zero $n$-dimensional measure, $N_{2}=N_{2}^{*}$ and $N_{3}=N_{3}^{*}$. By (3.3) we have for $i, j<n$

$$
\frac{\left(\frac{\partial H}{\partial u_{i}}\right)_{u}-\left(\frac{\partial H}{\partial u_{i}}\right)_{u^{(0)}}}{u_{j}}=\frac{2}{u_{j}|M|}\left(\int_{N_{3}} x_{i} d V_{x}^{n}-\int_{N_{2}} x_{i} d V_{x}^{n}\right) .
$$

We will calculate the limit of (3.5) as either $u_{j} \rightarrow 0+$ or $u_{j} \rightarrow 0-$. In either case for $x \in N_{3}, x_{j} u_{j} \geqq 0, x_{n} \leqq 0$ and for $x \in N_{2}, x_{j} u_{j} \geqq 0, x_{n} \geqq 0$. For $-\pi / 2<v_{n}<\pi / 2$, let the hyperplane $x_{n}=\left(\tan v_{n}\right) x_{j}$ intersect $M$ in $M^{+}\left(v_{n}\right)$ for $x_{n} \geqq 0$ and in $M^{-}\left(v_{n}\right)$ for $x_{n} \leqq 0$. Also the volume element $d V_{x}^{n-1}$ of this hyperplane is

$$
d V_{x}^{n-1}=d x_{1} \cdots d x_{n-1} \sec v_{n} .
$$

We introduce new coordinates $v_{1}, \cdots, v_{n}$ by $x_{i}=v_{i}$ for $i=1, \cdots, n-1$ and $x_{n}=v_{j} \tan v_{n}$ which uniquely define the $v_{i}$ with $-\pi / 2<v_{n}<\pi / 2$ for all $x$ for which $x_{j} \neq 0$. The Jacobian $J$ of this transformation is $J=v_{j} \sec ^{2} v_{n}$. Also define $\alpha, 0 \leqq \alpha<\pi / 2$, by $u_{n}^{(0)} \tan \alpha=\left|u_{j}\right|$. Then $|\boldsymbol{J}| / u_{j}= \pm v_{j} \sec ^{2} v_{n} / u_{n}^{(0)} \tan \alpha$ with the plus sign for $x \in N_{3}$ and the minus sign for $x \in N_{2}$. The difference quotient (3.5) is, consequently, given by

$$
\frac{2}{u_{n}^{(n)}|M| \tan \alpha}\left[\int_{0}^{\alpha} \sec v_{n}\left(\int_{\mathcal{M}^{-}\left(v_{n}\right)} v_{i} v_{j} d V_{v}^{n-1}\right) d v_{n}\right.
$$




$$
\left.+\int_{0}^{\infty} \sec v_{n}\left(\int_{\left.M^{+}+v_{n}\right)} v_{i} v_{j} d V_{v}^{n-1}\right) d v_{n}\right]
$$

and since the integrands are continuous functions of $v_{n}$ by assumption (b) we have

$$
H_{i j}\left(u^{(0)}\right)=\frac{\partial^{2} H\left(u^{(0)}\right)}{\partial u_{i} \partial u_{j}}=\frac{2}{u_{n}^{(0)}|M|} \int_{M(u(0))} x_{i} x_{j} d V_{x}^{n-1},(i, j<n) .
$$

Now let $H^{(i)}(u)$ be the supporting function (3.1) for the set $M_{i}$, $i=1, \cdots, n-1$. Set $H=\lambda_{1} H^{(1)}+\cdots+\lambda_{n} H^{(n-1)}$, then $D_{n-1}(H)$ is defined as the sum of all principal $(n-1)$ rowed minors of the matrix $H_{i j}$ (with components evaluated for a unit vector) and is a homogeneous polynomial of degree $(n-1)$ in the $\lambda_{i}$. (See [4, p. 59] or [2, pp. 45-46].) The quantity $D\left(H^{(1)}, \cdots, H^{(n-1)}\right)$ denotes the factor of $\lambda_{1} \cdots \lambda_{n-1}$ in $D_{n-1}(H)$ divided by $(n-1)$ !. If we calculate $(3.7)$ for each of the $H^{(i)}$ using the same standard coordinates we have, because of (3.4),

$$
D\left(H^{(1)}, \cdots, H^{(n-1)}\right)=\frac{1}{(n-1) !} \sum_{\left(i_{1} \cdots n_{n-1}\right)}\left|\begin{array}{ccc}
H_{11}^{\left(i_{1}\right)} & \cdots & H_{n(n-1)}^{\left(i_{n}\right)} \\
\vdots & & \vdots \\
H_{(n-1) 1}^{\left(i_{1}\right)} & \cdots & H_{(n-1)(n-1)}^{\left(i_{n}\right)}
\end{array}\right| .
$$

In the same way as we derived (2.10), we find for any $u \in \Omega_{n}$,

$$
\begin{aligned}
& D\left(H^{(1)}, \cdots, H^{(n-1)}\right) \\
= & \frac{(n-1) ! 2^{n-1}}{\left|M_{1}\right| \cdots\left|M_{n-1}\right|} \int_{M_{1}(u)} \cdots \int_{M_{n-1}(u)} T^{2}\left(z, p_{1}, \cdots, p_{n-1}\right) d V_{p_{1}}^{n-1} \cdots d V_{p_{n-1}}^{n-1} .
\end{aligned}
$$

By comparison with (2.10) we observe that

$$
D\left(H^{(1)}, \cdots, H^{(n-1}\right)=\frac{2^{n-1}\left|M_{1}(u)\right| \cdots\left|M_{n-1}(u)\right|}{\left|M_{1}\right| \cdots\left|M_{n-1}\right|} D\left(q_{1}, \cdots, q_{n-1}\right)
$$

where $q_{i}$ is the quadratic from associated with the Fenchel momental ellipsoid of $M_{i}(u)$ in the $(n-1)$-dimensional space $L(u)$.

From (3.9), we may give an integral interpretation of an elementary symmetric function $\left\{R_{1} \cdots R_{m}\right\}$ of the principal radii of curvature of the centroid surface of $M$. With $H$ given by (3.1) we have for $m=1, \cdots$, $n-1$ (see $[4$, p. 63]),

$$
\left\{R_{1} \cdots R_{m}\right\}=\left(\begin{array}{c}
\mathrm{n}-1 \\
\mathrm{~m}
\end{array}\right) D(\underbrace{|u|, \cdots,|u|}_{n-m-1}, \underbrace{H, \cdots, H}_{m})=D_{m}(H) .
$$

Set $M=M_{1}=\cdots=M_{m}$ and $U_{n}=M_{m+1}=\cdots=M_{n-1}$. Since

$$
\frac{1}{\left|U_{n}\right|} \int_{U_{n}}|u \cdot x| d V_{x}=\frac{2 \pi_{n-1}}{(n+1) \pi_{n}}|u| \text {, }
$$


we obtain

$$
\begin{aligned}
& {\left[\frac{2 \pi_{n-1}}{(n+1) \pi_{n}}\right]^{n-m-1} D_{m}(H)=\left(\begin{array}{c}
n-1 \\
m
\end{array}\right) \frac{(n-1) ! 2^{n-1}}{|M|^{m} \pi_{n}^{n-m-1}}} \\
& \cdot \underbrace{\int_{M(u)} \int_{M(u)}}_{m} \underbrace{\int_{\Pi_{n}(u)}}_{n-m-1} \int_{U_{n}(u)} T^{2}\left(z, p_{1}, \cdots, p_{n-1}\right) d V_{p_{1}}^{n-1} \cdots d V_{p_{n-1}^{n-1}}^{n-1} .
\end{aligned}
$$

By integrating successively over the $U_{n}(u)$ and using (2.3) applied to the appropriate dimensions we obtain

$$
\left\{R_{1} \cdots R_{m}\right\}=\frac{m ! 2^{m}}{|M|^{m}} \int_{M(u)} \cdots \int_{M(u)} T^{2}\left(z, p_{1}, \cdots, p_{m}\right) d V_{p_{1}}^{n-1} \cdots d V_{p_{m}}^{n-1}
$$

for $m=1, \cdots, n-1$.

We may also give an interpretation of each individual principal radius of curvature. First we show:

(3.14) The Dupin indicatrix of the centroid surface of $M(w r t z)$ at the point of contact $y$ of the tangent plane in the direction $u$ is homothetic to the Fenchel ellipsoid (wrt $z$ ) of $M(u)$ in the space $L(u)$.

A central affinity sends homothetic figures in parallel hyperplanes into homothetic figures. Due to the affine nature of Fenchel ellipsoids and centroid surfaces, we need only show that if the Fenchel ellipsoid of $M(u)$ is a sphere, then the Dupin indicatrix at $y$ is a sphere. However, this follows at once from (2.4) and the representation (3.7) in standard coordinates since the principal radii of curvature $R_{i}$ must satisfy

$$
\left|\begin{array}{ccc}
H_{11}-R & \cdots & H_{1 n} \\
\vdots & \vdots \\
H_{n 1} & \cdots & H_{n n}-R
\end{array}\right|=0
$$

where $H_{i j}$ are evaluated for a unit vector. (See [4, p. 61].)

Now, let the line through $z$, parallel to the $i$ th principal direction of the centroid surface at $y$, be normal to the $(n-2)$ space $L_{n-2}$ through $z$ in $L(u)$. Then $R_{i}$ is given by

$$
R_{i}=\frac{2}{|M|} I\left(M(u), L_{n-2}\right)
$$

where $I\left(M(u), L_{n-2}\right)$ is the second moment, in $L(u)$, of $M(u)$ with respect. to $L_{n-2}$.

Returning to the $(n-1)$ bodies $M_{1}, \cdots, M_{n-1}$ for which we obtained (3.9), let $H^{(n)}(u)$ be the supporting function (3.1) corresponding to any bounded closed set $M_{n}$ with positive volume. Then (see [2, p. 46]),

$$
V\left(K_{1}^{*}, \cdots, K_{n}^{*}\right)=n^{-1} \int_{\Omega_{n}} H^{(n)} D\left(H^{(1)}, \cdots, H^{(n-1)}\right) d \omega_{u}^{n},|u|=1,
$$


where $V\left(K_{1}^{*}, \cdots, K_{n}^{*}\right)$ is the mixed volume of $K_{1}^{*}, \cdots, K_{n}^{*}$. Using (3.9), (3.15), (3.1) and the integration technique of Busemann in [1] where it is shown that

$$
d V_{p_{1}}^{n} \cdots d V_{p_{n-1}}^{n}=(n-1) ! T\left(z, p_{1}, \cdots, p_{n-1}\right) d V_{p_{1}}^{n-1} \cdots d V_{p_{n-1}}^{n-1} d \omega_{u}^{n},
$$

we obtain

$$
\begin{aligned}
& V\left(K_{1}^{*}, \cdots, K_{n}^{*}\right) \\
& \quad=\frac{2^{n}}{\left|M_{1}\right| \cdots\left|M_{n}\right|} \int_{M_{1}} \cdots \int_{M_{n}} T\left(z, p_{1}, \cdots, p_{n}\right) d V_{p_{1}}^{n} \cdots d V_{p_{n}}^{n} .
\end{aligned}
$$

Since both sides of (3.16) vary continuously with the $M_{i}$, we may extend this result to any $n$ bounded and closed sets $M_{i}$ with $\left|M_{i}\right|>0$. Briefly, we may assume $z \in M_{i}$ and let $\varepsilon_{j}>0$ be a sequence such that $\varepsilon_{j} \rightarrow 0$. A covering of open spheres of radius $\varepsilon_{j}$ with centers in $M_{i}$ may be reduced to a finite covering since $M_{i}$ is compact. Conditions (a) and (b) are then satisfied for the closure of such a finite covering and the extention of (3.16) follows.

There is an alternate proof of (3.16) which proceeds directly from (3.1). We did not resort to this at the outset since the intervening results are of interest in themselves. Briefly, the alternate proof is as follows: We approximate the $H^{(i)}(u)$ of (3.1) by

$$
E^{(i, k)}(u)=\frac{1}{\left|M_{i}\right|} \sum_{j=1}^{k}\left|u \cdot x^{(j)}\right| \Delta V_{j}^{n}
$$

such that $E^{(i, k)} \rightarrow H^{(\imath)}$ as $k \rightarrow+\infty$. Now $|u \cdot x|$ is the supporting function of the segment $\bar{x}$ with end-points $x$ and $-x$. Also, by induction, one shows that

$$
V\left(\bar{x}^{(1)}, \cdots, \bar{x}^{(n)}\right)=2^{n} T\left(z, x^{(1)}, \cdots, x^{(n)}\right) .
$$

The function $E^{(i, k)}$ is the supporting function of the linear combination

$$
E_{(i, k)}=\frac{1}{\left|M_{i}\right|} \sum_{j=1}^{k} \bar{x}^{(j)} \Delta V_{j}^{n}
$$

For $\lambda_{j}>0$ the linear combination $E_{k}=\lambda_{1} E_{(1, k)}+\cdots+\lambda_{n} E_{(n, k)}$ may also be expressed as a linear combination of the $n k$ segments $\bar{x}^{\left(j_{i}\right)}$. Expressing the volume of $E_{k}$ as a polynomial in the $\lambda_{i}$ in two ways we have by comparing the coefficient of $\lambda_{1} \cdots \lambda_{n}$

$$
\begin{aligned}
& V\left(E_{(1, k)}, \cdots, E_{(n, k)}\right) \\
& =\frac{2^{n}}{\left|M_{1}\right| \cdots\left|M_{n}\right|} \sum_{j_{1}=1}^{k} \cdots \sum_{j_{n}=1}^{k} T\left(z, x^{\left(j_{1}\right)}, \cdots, x^{\left(j_{n}\right)}\right) \Delta V_{j_{1}}^{n} \cdots \Delta V_{j_{n}}^{n}
\end{aligned}
$$

and (3.16) follows in the limit as $k \rightarrow+\infty$. 
The formula (3.16) may be substituted into inequalities of mixed volumes to yield inequalities of the integrals. Since the number of times a component appears on each side of a mixed volume inequality is always the same, the coefficient on the righthand side of (3.16) cancels leaving, as in (2.16), inequalities among the integrals only. However, in this case when the uniqueness theorem (4.1) applies, the condition for equality may be passed through the $K_{i}^{*}$ to the $M_{i}$.

In [1, p. 11], Busemann shows that if $M$ is a nondegenerate convex body, then

$$
\int_{M} \cdots \int_{M} T\left(z, p_{1}, \cdots, p_{n}\right) d V_{p_{1}} \cdots d V_{p_{n}} \geqq \frac{2}{(n+1) !} \frac{\pi_{n+1}^{n-1}}{\pi_{n}^{n+1}}|M|^{n+1}
$$

with equality only if $M$ is an ellipsoid with center $z$. We define the expanded centroid body $K$ of $M$ to be the dilation of $K^{*}$ about $z$ by the factor $(n+1) \pi_{n} / 2 \pi_{n-1}$. By (3.12), we see that this is the factor which dilates the centroid body of an ellipsoid with center $z$ into coincidence with the ellipsoid.

From (3.16) we obtain a reinterpretation of (3.17) by observing the identity $n ! \pi_{n} \pi_{n-1}=2^{n} \pi^{n-1}$ :

(3.18) If $K$ is the expanded centroid body of a nondegenerate convex body $M$, then $|K| \geqq|M|$ with equality only if $M$ is an ellipsoid with center $z$.

The convexity of $M$ is not an essential feature in (3.18) and the Steiner symmetrization used to prove (3.17) may be extended to include nonconvex sets.

Using the expanded centroid bodies $K_{i}$ of $M_{i}$, we may write (3.16) as

$$
\begin{aligned}
& \left|M_{1}\right| \cdots\left|M_{n}\right| V\left(K_{1}, \cdots, K_{n}\right) \\
& \quad=\frac{(n+1) ! \pi_{n}^{n+1}}{2 \pi_{n+1}^{n-1}} \int_{M_{1}} \cdots \int_{M_{n}} T\left(z, p_{1}, \cdots, p_{n}\right) d V_{p_{1}}^{n} \cdots d V_{p_{n}}^{n}
\end{aligned}
$$

and if we define $K_{i}$ to be the point $z$ if $\left|M_{i}\right|=0$ then (3.19) holds for any bounded closed sets $M_{i}$.

Substituting (3.19) into (1.1) we have

(3.20) THEOREM. If $K_{i}(u)$ is the expanded centroid body of $M_{i}(u)$ in the $(n-1)$-dimensional space $L(u)$, then

$$
\begin{aligned}
&\left|M_{1}\right| \cdots\left|M_{n-1}\right| \\
& \quad=\frac{1}{n} \frac{\pi_{n}^{n-2}}{\pi_{n-1}^{n}} \int_{\Omega_{n}}\left|M_{1}(u)\right| \cdots\left|M_{n-1}(u)\right| V\left(K_{1}(u), \cdots, K_{n-1}(u)\right) d \omega_{u}^{n} .
\end{aligned}
$$

The inequality $V^{n-1}\left(K_{1}(u), \cdots, K_{n-1}(u)\right) \geqq\left|K_{1}(u)\right| \cdots\left|K_{n-1}(u)\right|$ (see $[2$, p. 50]) and (3.18) reproduces (1.3). 
There are two special cases of (3.20) of particular geometric interest. First, set $M=M_{1}=\cdots=M_{n-1}$, then

$$
|M|^{n-1}=\frac{1}{n} \frac{\pi_{n}^{n-2}}{\pi_{n-1}^{n}} \int_{\Omega_{n}}|M(u)|^{n-1}|K(u)| d \omega_{u}^{n} .
$$

Next, for $n \geqq 3$, set $M=M_{1}=\cdots=M_{n-2}, M_{n-1}=U_{n}$, then

$$
|M|^{n-2}=\frac{1}{n(n-1)} \frac{\pi_{n}^{n-3}}{\pi_{n-1}^{n-1}} \int_{\Omega_{n}}|M(u)|^{n-2} S(K(u)) d \omega_{u}^{n}
$$

where $S(K(u))$ is the surface area of $K(u)$ in the space $L(u)$.

4. Uniqueness theorems. In order for $K^{*}$ to determine $M$, additional restrictions on $M$ are necessary as may be seen by consideration of a set $M$ bounded by two concentric spheres.

(4.1) Theorem. Suppose $M_{i}(i=1,2)$ can be represented in polar coordinates by $0 \leqq r \leqq \rho_{2}(u), u \in \Omega_{n}$ where $\rho_{i}(u)$ is an even, i.e., $\rho_{i}(u)=$ $\rho_{i}(-u)$, continuous function on $\Omega_{n}$. If the centroid surface of $M_{1}($ wrt $z)$ is identical to the centroid surface of $M_{2}($ urt $z)$, then $M_{1}$ and $M_{2}$ are identical.

(4.2) Theorem. Suppose $M_{i}(i=1,2)$ have the same representation as in (4.1). If $\left|M_{1}(u)\right|=\left|M_{2}(u)\right|$ for all $u \in \Omega_{n}$, then $M_{1}$ and $M_{2}$ are identical.

The latter theorem is a result, for $n=3$, of $P$. Funk [6].

We first prove (4.1). From (3.1) and the assumption on the representation of $M_{i}$ we have

$$
H^{(i)}(u)=\frac{1}{(n+1)\left|M_{i}\right|} \int_{\Omega_{n}}|u \cdot \tau| \rho_{i}^{n+1}(\tau) d \omega_{\tau}^{n},|\tau|=1 .
$$

Consequently, (4.1) follows from the uniqueness of the solution of an integral equation of the first kind. Namely:

(4.3) TheOREM. Let $h(\tau)$ be an even, continuous function on $\Omega_{n}$. If for unit vectors $u$ and $\tau$

$$
\int_{a_{n}}|u \cdot \tau| h(\tau) d \omega_{\tau}^{n}=0
$$

for all $u \in \Omega_{n}$, then $h(\tau)$ vanishes identically.

The result (4.3) is well known for $n=2,3$ and the recent extension of surface harmonics to $n$-dimensions, in particular the Funk-Hecke theorem, enables one to prove (4.3) for all $n$. There are two steps in the following proof (which applies for $n \geqq 3$ ). First, from the com- 
pleteness [5, p. 241] it suffices to show that

$$
\int_{\Omega_{n}} S_{m}(\tau) h(\tau) d \omega_{\tau}^{n}=0
$$

for all the linearly independent surface harmonics $S_{m}(\tau)$ of degree $m$ and for $m=0,1,2, \cdots$. Since $h(\tau)$ is an even function we need only to consider, now, even $m$. Next, from the Funk-Hecke theorem [5, pp. 247-248] we have

$$
\int_{\Omega_{n}}|u \cdot \tau| S_{m}(u) d \omega_{u}^{n}=\lambda_{m} S_{m}(\tau)
$$

where

$$
\lambda_{m}=\frac{(4 \pi)^{\nu} m ! \Gamma(\nu)}{(m+2 \nu-1) !} \int_{-1}^{1}|x| C_{m}^{\nu}(x)\left(1-x^{2}\right)^{\nu-1 / 2} d x
$$

and $\nu=(n-2) / 2 \geqq 1 / 2$. Thus, we need only to verify that $\lambda_{m} \neq 0$ for $m=0,2,4, \cdots$. For $m=0, C_{0}^{\nu}(x)=1$ and $\lambda_{0} \neq 0$. For $m>0$,

$$
C_{m}^{\nu}(x)=a_{m, \nu}\left(1-x^{2}\right)^{-\nu+1 / 2} \frac{d^{m}}{d x^{m}}\left[\left(1-x^{2}\right)^{m+\nu-1 / 2}\right]
$$

where $a_{m, \nu} \neq 0$. See [5, p. 236] for the explicit expression of the coefficient $a_{m, \nu}$. Thus the integral in (4.4) is

$$
I_{m, \nu}=2 a_{m, \nu} \int_{0}^{1} x \frac{d^{m}}{d x^{m}}\left[\left(1-x^{2}\right)^{m+\nu-1 / 2}\right] d x
$$

and using integration by parts

$$
I_{m, \nu}=2 a_{m, \nu}(-1)^{\frac{m-2}{2}}(m-2) !\left(\begin{array}{c}
m+\nu-1 / 2 \\
\frac{m}{2}-1
\end{array}\right) \neq 0
$$

for $m=2,4,6, \ldots$ which completes the proof.

The result (4.2) is clearly a consequence of the following spherical integration theorem.

(4.5) Let $f(\tau)$ be a continuous even function defined on $\Omega_{n}$. If

$$
\int_{\Omega_{n}(u)} f(\tau) d \omega_{\tau}^{n-1}=0
$$

for all $u \in \Omega_{n}$, then $f(\tau)$ vanishes identically.

A proof of (4.5) for $n=3$ can be found in [4, pp. 136-138]. However, a proof for all $n \geqq 3$ is easily obtained from (4.1). To see this, set $g(\tau)=f(\tau)-[\min f(\tau)]+1>0$. Let $\rho(\tau)=[g(\tau)]^{1 / n+1)}$ and let $M$ be the set whose polar coordinates satisfy $0 \leqq r \leqq \rho(\tau), \tau \in \Omega_{n}$. Using (3.13) for $m=1$, the sum of the principal radii of curvature of the centroid 
surface of $M$ wrt $z$ may be expressed, in this case, by

$$
R_{1}+\cdots+R_{n-1}=\frac{2}{(n+1)|M|} \int_{\Omega_{n}(u)} g(\tau) d \omega_{\tau}^{n-1}
$$

and, by hypothesis, this is a positive constant for $u \in \Omega_{n}$. However, this implies (see [4, pp. 117-118]) that the centroid surface is a sphere and by (4.1), $M$ is a solid sphere and $g(\tau)$ is a constant which completes the proof.

\section{REFERENCES}

1. H. Busemann, Volume in terms of concurrent cross-sections, Pacific J. Math., 3 (1953). 1-12.

2. — Convex Surfaces, Interscience Publishers, New York, 1958.

3. H. Busemann and E. G. Straus, Area and normality, Pacific J. Math., 10 (1960), 35-72.

4. T. Bonnesen and W. Fenchel, Theorie der konvexen Körper, Chelsea, New York, 1948.

5. A. Erdélyi, et al., Higher transcendental functions, Bateman Manuscript Project, vol.

2, New York, 1953.

6. P. Funk, Über Flächen mit lauter geschlossenen geodätischen Linien, Math. Ann., 74 (1913), 278-300.

Applied Mathematics, Lockheed Missiles and Space Division, Sunnyvale, California 



\section{PACIFIC JOURNAL OF MATHEMATICS}

\section{EDITORS}

\author{
RaLPh S. Phillips \\ Stanford University \\ Stanford, California \\ F. H. BRowNELL \\ University of Washington \\ Seattle 5 , Washington
}

A. L. Whiteman

University of Southern California

Los Angeles 7, California

L. J. Paige

University of California

Los Angeles 24, California

\author{
E. F. BECKENBACH \\ T. M. CHERRY
}

\author{
ASSOCIATE EDITORS

$\begin{array}{lll}\text { D. DERRY } & \text { H. L. ROYDEN } & \text { E. G. STRAUS } \\ \text { M. OHTSUKA } & \text { E. SPANIER } & \text { F. WOLF }\end{array}$

\section{SUPPORTING INSTITUTIONS}

\author{
UNIVERSITY OF BRITISH COLUMBIA \\ CALIFORNIA INSTITUTE OF TECHNOLOGY \\ UNIVERSITY OF CALIFORNIA \\ MONTANA STATE UNIVERSITY \\ UNIVERSITY OF NEVADA \\ NEW MEXICO STATE UNIVERSITY \\ OREGON STATE COLLEGE \\ UNIVERSITY OF OREGON \\ OSAKA UNIVERSITY \\ UNIVERSITY OF SOUTHERN CALIFORNIA
}

\author{
STANFORD UNIVERSITY \\ UNIVERSITY OF TOKYO \\ UNIVERSITY OF UTAH \\ WASHINGTON STATE COLLEGE \\ UNIVERSITY OF WASHINGTON \\ AMERICAN MATHEMATICAL SOCIETY \\ CALIFORNIA RESEARCH CORPORATION \\ HUGHES AIRCRAFT COMPANY \\ SPACE TECHNOLOGY LABORATORIES \\ NAVAL ORDNANCE TEST STATION
}

Mathematical papers intended for publication in the Pacific Journal of Mathematics should be typewritten (double spaced), and the author should keep a complete copy. Manuscripts may be sent to any one of the four editors. All other communications to the editors should be addressed to the managing editor, L. J. Paige at the University of California, Los Angeles 24, California.

50 reprints per author of each article are furnished free of charge; additional copies may be obtained at cost in multiples of 50 .

The Pacific Journal of Mathematics is published quarterly, in March, June, September, and December. The price per volume (4 numbers) is $\$ 12.00$; single issues, $\$ 3.50$. Back numbers are available. Special price to individual faculty members of supporting institutions and to individual members of the American Mathematical Society: $\$ 4.00$ per volume; single issues, $\$ 1.25$.

Subscriptions, orders for back numbers, and changes of address should be sent to Pacific Journal of Mathematics, 103 Highland Boulevard, Berkeley 8, California.

Printed at Kokusai Bunken Insatsusha (International Academic Printing Co., Ltd.), No. 6, 2-chome, Fujimi-cho, Chiyoda-ku, Tokyo, Japan.

\section{PUBLISHED BY PACIFIC JOURNAL OF MATHEMATICS, A NON-PROFIT CORPORATION}

The Supporting Institutions listed above contribute to the cost of publication of this Journal, but they are not owners or publishers and have no responsibility for its content or policies.

Reprinted 1966 in the United States of America 


\section{Pacific Journal of Mathematics}

\section{Vol. 11, No. 4}

A. V. Balakrishnan, Prediction theory for Markoff processes . . . . . . . . . . 1171

Dallas O. Banks, Upper bounds for the eigenvalues of some vibrating systems . . . . 1183

A. Białynicki-Birula, On the field of rational functions of algebraic groups ...... 1205

Thomas Andrew Brown, Simple paths on convex polyhedra .............. 1211

L. Carlitz, Some congruences for the Bell polynomials . . . . . . . . . . . . 1215

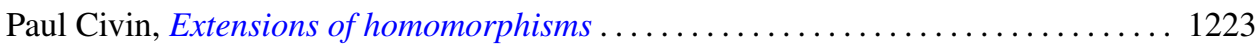

Paul Joseph Cohen and Milton Lees, Asymptotic decay of solutions of differential

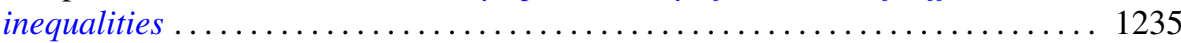

István Fáry, Self-intersection of a sphere on a complex quadric . . . . . . . . . . 1251

Walter Feit and John Griggs Thompson, Groups which have a faithful representation

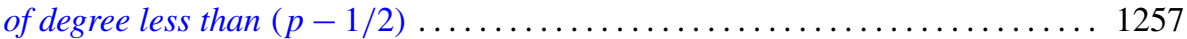

William James Firey, Mean cross-section measures of harmonic means of convex

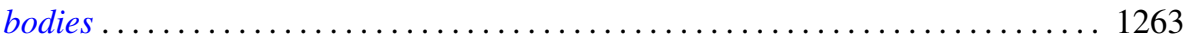

Avner Friedman, The wave equation for differential forms . . . . . . . . . . 1267

Bernard Russel Gelbaum and Jesus Gil De Lamadrid, Bases of tensor products of

Banach spaces ................................... 1281

Ronald Kay Getoor, Infinitely divisible probabilities on the hyperbolic plane . . . . 1287

Basil Gordon, Sequences in groups with distinct partial products . . . . . . . . . . . . 1309

Magnus R. Hestenes, Relative self-adjoint operators in Hilbert space . . . . . . . . . 1315

Fu Cheng Hsiang, On a theorem of Fejér ......................... 1359

John McCormick Irwin and Elbert A. Walker, On N-high subgroups of Abelian

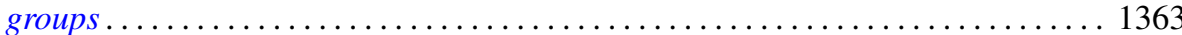

John McCormick Irwin, High subgroups of Abelian torsion groups . . . . . . . . . 1375

R. E. Johnson, Quotient rings of rings with zero singular ideal . . . . . . . . . . . 1385

David G. Kendall and John Leonard Mott, The asymptotic distribution of the time-to-escape for comets strongly bound to the solar system ...

Kurt Kreith, The spectrum of singular self-adjoint elliptic operators ....

Lionello Lombardi, The semicontinuity of the most general integral of the calculus of variations in non-parametric form ................................

Albert W. Marshall and Ingram Olkin, Game theoretic proof that Chebyshev inequalities are sharp

Wallace Smith Martindale, III, Primitive algebras with involution . . William H. Mills, Decomposition of holomorphs ..............

James Donald Monk, On the representation theory for cylindric algebras . . . . . . 1447

Shu-Teh Chen Moy, A note on generalizations of Shannon-McMillan theorem . . . . 1459

Donald Earl Myers, An imbedding space for Schwartz distributions . .

John R. Myhill, Category methods in recursion theory .........

Paul Adrian Nickel, On extremal properties for annular radial and circular slit mappings of bordered Riemann surfaces

Edward Scott O'Keefe, Primal clusters of two-element algebras . .

Nelson Onuchic, Applications of the topological method of Wazewski to certain

problems of asymptotic behavior in ordinary differential equations ...

Peter Perkins, A theorem on regular matrices................

Clinton M. Petty, Centroid surfaces .... 